Bundesgesundheitsbl $2017 \cdot 60: 556-562$

DOI 10.1007/s00103-017-2537-6

Online publiziert: 8. März 2017

(c) Springer-Verlag Berlin Heidelberg 2017

CrossMark

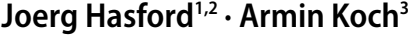 \\ ${ }^{1}$ Institut für Medizinische Informationsverarbeitung, Biometrie und Epidemiologie, Ludwig-Maximilians- \\ Universität München, München, Deutschland \\ ${ }^{2}$ Arbeitskreis Medizinischer Ethik-Kommissionen in der BRD e. V., München, Deutschland \\ ${ }^{3}$ Institut für Biometrie, Medizinische Hochschule Hannover, Hannover, Deutschland
}

\title{
Ethische Aspekte der klinischen Prüfung bei seltenen Erkrankungen
}

\section{Hintergrund}

Nach vielen Jahren der Vernachlässigung stehen seltene Erkrankungen nun seit über 20 Jahren auch im Fokus gesundheitspolitischer Aufmerksamkeit. Im Jahr 1999 verabschiedeten das Europäische Parlament und der Europäische Rat die Verordnung EG Nr. 141/2000 über Arzneimittel für seltene Erkrankungen. Diese Verordnung definiert seltene Erkrankungen in Artikel 3 als „... ein Leiden von dem in der Gemeinschaft nicht mehr als fünf von zehntausend Personen betroffen sind." Es wird geschätzt, dass in Deutschland mehr als 4 Mio. Menschen an einer seltenen Erkrankungen leiden und also, obwohl jede einzelne Erkrankung selten ist, insgesamt eine beachtliche Anzahl von Menschen von einer seltenen Erkrankung betroffen ist. Die weltweit rund 8000 verschiedenen seltenen Erkrankungen machen zusammen ein Viertel aller bekannten Krankheiten aus [1]. „Seltene Erkrankungen weisen einige Besonderheiten auf: Dazu zählen vordringlich die geringe Zahl an Patientinnen und Patienten mit einer seltenen Erkrankung, eine die Durchführung von Studien erschwerende überregionale Verteilung und eine geringe Anzahl von räumlich verteilten Expertinnen und Experten, die an ei-

Wann immer im Text Personen oder Funktionen männlich adressiert werden, sind beide Geschlechter gemeint. Die Ausführungen von J. Hasford geben nicht notwendigerweise die Auffassungen des Arbeitskreises Medizinischer Ethik-Kommissionen wieder. ner seltenen Erkrankung arbeiten." [1]. Der Nationale Aktionsplan für Deutschland identifizierte darüber hinaus noch folgende Schwierigkeiten für klinische Prüfungen in diesem Feld: „Die Durchführung von klinischen Studien in Indikationen von seltenen Erkrankungen, und somit die zielgerichtete Entwicklung von neuen Therapieformen, ist im Vergleich zu häufigeren Krankheitsbildern schwierig. Insbesondere in den drei Bereichen Finanzierung, Regularien sowie statistische Planung und Auswertung bei seltenen Erkrankungen sind die Erfordernisse komplex und erfordern zusätzliches Detailwissen, das von den einzelnen Einrichtungen nicht vorgehalten werden kann." [1]. Ziel unseres Beitrages ist es, eine Einführung in einige Grundlagen der ethischen Bewertung des Antrags auf klinische Prüfung durch die zuständige Ethik-Kommission zu geben und Prinzipien der Bewertung des Versuchsplans aus biometrischer Sicht zu diskutieren, da die ethische Vertretbarkeit einer Studie ein gutes wissenschaftliches und methodisches Konzept voraussetzt.

\section{Aufgaben der Ethik-Kommission}

Die ersten Ethik-Kommissionen für die Beratung medizinischer Forschungsprojekte in Deutschland wurden 1973 von Vertretern medizinischer Fakultäten gegründet. Ihr Impetus galt der freiwilligen Beratung von ärztlichen Kollegen, die Forschungsprojekte durchführen wollten, die sie selbst als ethisch konflikt- trächtig ansahen, und daher kollegialen Rat bei Experten suchten. 1983 gab es bereits so viele Ethik-Kommissionen an medizinischen Fakultäten und Landesärztekammern, dass der Arbeitskreis Medizinischer Ethik-Kommissionen in der Bundesrepublik Deutschland gegründet werden konnte. 1995 wurden Ethik-Kommissionen erstmalig im Arzneimittelgesetz (AMG) erwähnt. Seit 2004 ist mit dem ergänzten $\$ 40$ (1) die ,zustimmende Bewertung der zuständigen Ethik-Kommission zwingende Voraussetzung für die Durchführung einer klinischen Prüfung. Damit sind die Ethik-Kommissionen schlechthin $\mathrm{zu}$ "der“ Institution für den Patientenund Probandenschutz in der klinischen Prüfung geworden. Da die klinische Prüfung in $\$ 4$ (23) AMG sehr breit definiert wurde, muss praktisch alle Arzneimittelforschung in Deutschland vorab der zuständigen Ethik-Kommission vorgelegt werden. Der rechtliche Rahmen und der Umfang dieser Bewertung ist durch $\$$ 40-42a AMG, die Good-ClinicalPractice-Verordnung und die RICHTLINIE 2001/20/EG DES EUROPÄISCHEN PARLAMENTS UND DES RATES vom 4. April 2001 zur Angleichung der Rechtsund Verwaltungsvorschriften der Mitgliedstaaten über die Anwendung der guten klinischen Praxis bei der Durchführung von klinischen Prüfungen mit Humanarzneimitteln festgelegt. Artikel 6 der Richtlinie 2001/20/EG legt den Prüfumfang detailliert fest: „Die Ethik-Kommission berücksichtigt bei der Ausarbeitung ihrer Stellungnahme 
insbesondere: a) die Relevanz der klinischen Prüfung und ihrer Planung, $b$ ) die Angemessenheit der in Artikel 3 Absatz 2 Buchstabe a) vorgeschriebenen Bewertung des erwarteten Nutzens und der erwarteten Risiken und die Begründetheit der Schlussfolgerungen, c) den Prüfplan, d) die Eignung des Prüfers und seiner Mitarbeiter, e) die Prüferinformation, f) die Qualität der Einrichtungen, g) die Angemessenheit und Vollständigkeit der zu erteilenden schriftlichen Auskünfte sowie das Verfahren im Hinblick auf die Einwilligung nach Aufklärung und die Rechtfertigung für die Forschung an Personen, die zur Einwilligung nach Aufklärung nicht in der Lage sind, was die spezifischen in Artikel 3 niedergelegten Einschränkungen anbelangt, h) die Vorschriften für Wiedergutmachung oder Entschädigung bei Schäden oder Todesfällen, die auf die klinische Prüfung zurückzuführen sind, i) jede Art von Versicherung oder Schadenersatz zur Deckung der Haftung des Prüfers und des Sponsors, j) die Beträge und die Modalitäten für die etwaige Vergütung oder Entschädigung für Prüfer und Prüfungsteilnehmer und die einschlägigen Elemente jedes zwischen dem Sponsor und der Prüfstelle vorgesehenen Vertrags, k) die Modalitäten für die Auswahl der Prüfungsteilnehmer." Der Antragsteller, der in rechtlicher Hinsicht für die Veranlassung, Organisation und Finanzierung der Studie verantwortlich ist und bei klinischen Prüfungen Sponsor genannt wird, muss der EthikKommission alle Unterlagen, die für diese umfangreiche Bewertung erforderlich sind, vorlegen. Einzelheiten dazu finden sich im Artikel 7 der GCP-V (GoodClinical-Practice-Verordnung), das formale Verfahren der Ethik-Kommission selbst ist dort weitgehend in Artikel 8 geregelt.

\section{Ethisch-rechtliche Aspekte bei der Bewertung}

Bei der Bewertung der Anträge in den Ethik-Kommissionen wird auf der Basis der wesentlichen Prinzipien und Konzepte der Ethik in der Heilkunde und der medizinischen Forschung die ethische, rechtliche und ärztliche Vertret- barkeit der Studie geprüft. Sowohl die deontologische Ethik, die von Hippokrates abgeleitet wird, wie die Prinzipienethik nach Beauchamp und Childress [2], aber auch die utilitaristische Ethik bzw. der Konsequentialismus, die auf J. Bentham und S. J. Mills zurückgehen, sind hier einschlägig. Im multidisziplinären Diskurs zwischen den Mitgliedern der Ethik-Kommission werden die geeigneten ethischen Prinzipien und Konzepte individuell auf die jeweilige Studie angewendet und ein Beschluss über die ärztliche, rechtliche und ethische Vertretbarkeit der Studie herbeigeführt. Das grundgesetzlich verbriefte Recht auf Würde und Freiheit sowie auf Leben und körperliche Unversehrtheit gebietet Respekt und Achtung der Autonomie der Versuchsperson. Daher bedarf jegliche Forschung mit Menschen dervollumfänglichen Aufklärung über Wesen, Tragweite und Bedeutung der Studie mit nachfolgender Einwilligung des Betroffenen oder seines gesetzlichen Vertreters. Das Nichtschadensprinzip fordert, dass alles getan werden muss, um die Risiken und Belastungen der klinischen Prüfung zu minimieren, und das Fürsorgeprinzip, dass das Wohlergehen der Versuchsperson zu fördern ist. Das Prinzip der Gerechtigkeit verlangt, dass die Risiken und Belastungen der klinischen Prüfung fair verteilt sind und findet $z$. B. bei der Bewertung von Anträgen zu gruppennütziger Forschung und bei Forschung in Entwicklungsländern oder mit vulnerablen Menschen Anwendung. Das Arzneimittelgesetz sieht spezifische Anforderungen für klinische Prüfungen mit Patienten, Minderjährigen, Notfallpatienten und für Studien mit ausschließlich gruppennütziger Zielsetzung vor. Unter gruppennützig wird eine Studie verstanden, bei der der Teilnehmer keine Chance auf einen eigenen gesundheitlichen Nutzen hat, sondern nur zukünftige Patienten, die an der gleichen Erkrankung leiden. Vor allem Studien zur Pharmakokinetik und Biomarkerstudien zählen zu dieser Kategorie.

Viele der seltenen Erkrankungen manifestieren sich bereits beim minderjährigen Patienten. Die klinische Prüfung mit Minderjährigen ist in den Artikeln 40 (4) und 41 (2) AMG eigens geregelt. Ohne hier auf alle Einzelheiten einzugehen, sollen doch 3 Anforderungen kurz genannt werden: Auch Minderjährige müssen ab dem frühestmöglichen Alter, d. h. spätestens mit der Schulreife, in den Prozess der Aufklärung und der Einwilligung, ihrer geistigen Reife entsprechend, einbezogen werden. Eine Einbeziehung in eine klinische Prüfung von Jugendlichen, d. h. ab einem Alter von 12-14 Jahren, gegen ihren erklärten Willen wird als nicht statthaft angesehen. Auch die Meinungsbekundungen Jüngerer sind zu beachten. Selbstverständlich müssen auch alle Sorgeberechtigten, d.h. in der Regel beide Eltern, zustimmen. Schließlich sind placebokontrollierte klinische Prüfungen mit Minderjährigen ohne Ausnahme nicht zulässig, denn das AMG fordert, dass das zu prüfende Arzneimittel nach den Erkenntnissen der medizinischen Wissenschaft angezeigt sein muss, bei dem Minderjährigen entweder Krankheiten zu erkennen, ihn vor Krankheiten zu schützen ( $\$ 40$ [4] AMG) oder um dessen Leben zu retten, seine Gesundheit wiederherzustellen oder sein Leiden zu erleichtern ( $\$ 41$ [2] AMG). Da Placebos gemäß Artikel 3 (3) der GCP$\mathrm{V}$ zu den Prüfpräparaten gehören und ihr therapeutischer Einsatz nach den Erkenntnissen der medizinischen Wissenschaft kaum jemals angezeigt sein dürfte, ergibt sich dieses für die Erkenntnisgewinnung und Studienplanung relevante Verbot.

Das AMG und die GCP-V sehen für das Genehmigungsverfahren klinischer Prüfungen durch Bundesoberbehörde und Ethik-Kommission für Anträge zur Forschung mit seltenen Erkrankungen keine Sonderregelungen vor. Auch die Neuregelung der klinischen Prüfung in der Europäischen Union (EU), die mit der Verordnung EU Nr. 536/2014 voraussichtlich im Oktober 2018 in Kraft treten wird, sieht keine Sonderregelungen für die klinische Prüfung mit Patienten vor, die an einer seltenen Erkrankung leiden. Wenn schon die rechtlichen Rahmenbedingungen wie AMG und in absehbarer Zukunft die EU-Verordnung Nr. 536/2014 keine Sonderregelungen für klinische Prüfungen vorsehen, kann es nicht überraschen, dass es diese auch nicht für die ethische Bewertung gibt. 
Dies liegt auch darin begründet, dass die ersten drei der vier zuvor genannten Prinzipien sich direkt aus den grundgesetzlich verbrieften Rechten ergeben und daher nicht abdingbar sind.

Da die Evidenzgenerierung zu Wirksamkeit und Verträglichkeit von Arzneimitteln und Therapien bei häufigen Erkrankungen vor der Zulassung über eine Vielzahl klinischer Studien der Phasen I-III läuft, die zusammengenommen große Stichproben erfordern, ergeben sich bei seltenen Erkrankungen erhebliche Probleme für klinische Prüfungen. Fallzahlen von 1500 und mehr Patienten, wie sie zum Zeitpunkt der behördlichen Zulassung häufig vorliegen, sind für seltene Erkrankungen meist unerreichbar. In den letzten Jahren hat es daher verstärkte Anstrengungen gegeben, Studiendesigns, statistische Auswertungsverfahren und Strategien zum Erkenntnisgewinn speziell für seltene Erkrankungen zu entwickeln [3-5]. Ziel dieser Bemühungen ist es, ein möglichst hohes Evidenzniveau für Wirksamkeit und Verträglichkeit von Therapien für Patienten mit seltenen Erkrankungen $\mathrm{zu}$ ermöglichen. Nur aussagekräftige klinische Forschung ist ethisch vertretbar. Da Ethik-Kommissionen daher die wissenschaftliche Qualität der Anträge prüfen müssen, auch um sicherzustellen, das alles getan wurde, um mit so wenig Patienten wie möglich $\mathrm{zu}$ einer validen Aussage zu kommen, soll im Folgenden näher auf die biometrischen Aspekte der Erkenntnisgewinnung bei seltenen Erkrankungen eingegangen werden.

\section{Biometrische Aspekte}

Der genaue Blick auf das Problem aus statistischer Sicht ist aus leicht nachvollziehbaren Gründen eher ernüchternd: Die Wirksamkeit einer neuen Therapie wird entweder durch den Nachweis der Überlegenheit gegenüber Placebo oder dem bisherigen Standard belegt bzw. durch die Vergleichbarkeit zum bisherigen Standard. Im Falle einer Studie zum Nachweis der Überlegenheit hängt die dafür erforderliche Patientenzahl von 2 Faktoren ab: von der Größe des Therapieeffektes und von der Variabilität der Zielgröße. Führt die neue Therapie zu einer deutlichen

Bundesgesundheitsbl 2017·60:556-562 DOI 10.1007/s00103-017-2537-6

(c) Springer-Verlag Berlin Heidelberg 2017

\section{J. Hasford · A. Koch \\ Ethische Aspekte der klinischen Prüfung bei seltenen Erkrankungen}

\section{Zusammenfassung}

Seltene Erkrankungen, an denen allein in Deutschland geschätzte 4 Mio. Menschen leiden, sind in den Blickpunkt des Interesses der Therapieforschung gerückt. Die klinische Prüfung von Arzneimitteln ist sehr detailliert in Gesetzen, Verordnungen und ethischen Konventionen geregelt, die keine Sonderregelungen für klinische Prüfungen bei seltenen Erkrankungen vorsehen. Somit gelten für die ethische Bewertung entsprechender Anträge die gleichen Standards wie für häufige Krankheiten. Dies macht auch Sinn, da ethische Standards bezüglich Aufklärung, Einwilligung und Studiendurchführung, die zudem meist im Recht kodifiziert sind, nicht von der Häufigkeit der Erkrankung abhängen können. Besondere Bedeutung im Kontext der ethischen Bewertung spielen biometrische Aspekte wie Studiendesign und statistische Auswertung, da nicht aussagekräftig angelegte klinische Forschung mit Menschen per se unethisch ist. Auf die Probleme der Fallzahlschätzung und von Pilotstudien wird näher eingegangen. Pilotstudien sollten nach Möglichkeit vermieden werden. Bei der Fallzahlschätzung sollte nicht von überoptimistischen Annahmen ausgegangen werden und keine Erhöhung der üblichen Irrtumswahrscheinlichkeit von $5 \%$ erfolgen.

Schlüsselwörter

Seltene Erkrankungen · Klinische Prüfung Ethik · Biometrie

\section{Ethical aspects of clinical trials in rare diseases}

\section{Abstract}

It is estimated that there are about four million people suffering from rare diseases in Germany. For roughly the last 20 years, there has been an increasing interest in therapeutic research for rare diseases. Drug research is highly regulated via numerous laws, regulations and ethical conventions that do not offer any waivers for clinical trials in rare diseases. Thus the ethical assessment of the clinical trial application for a rare disease is basically the same as for a common disease. As the ethical standards of clinical research, for example regarding informed consent, are derived from constitutional rights and have been codified in the German drug law, it is no surprise that they cannot depend on the frequency of a disease. A very important aspect of the ethical assessment is the biometric quality with regard to study design, sample size estimation and statistical analysis, as methodologically poor research with humans is per se unethical. Problems with sample size estimations and pilot studies will be addressed in more detail. Pilot studies should be avoided and sample size estimations should not assume overoptimistic effect sizes and should not increase the error probability beyond 5\% two-sided.

\section{Keywords}

Rare diseases · Clinical trials · Ethical assessment $\cdot$ Biometry
Verbesserung im Sinne eines größeren Therapieeffektes, ist eine geringere $\mathrm{Pa}$ tientenzahl für den formalen Nachweis der Wirksamkeit nötig. Die erforderliche Fallzahl wird zudem größer, wenn die gewählte Zielgröße variabler ist im Vergleich zu einer weniger variablen Therapieantwort der Patienten. Daher ist es ein wichtiges Ziel der Versuchsplanung die Variabilität der Zielgröße möglichst gering zu halten.

Im Mittel werden die Fallzahlanforderungen an Studien für seltene Erkrankungen nicht kleiner sein als bei Studi- en für häufige Erkrankungen, da nicht häufiger Medikamente mit einem dramatischen Therapieeffekt entwickelt werden können als dies bei konventionellen „häufigen“Erkrankungen der Fall ist. Zusätzlich wird die Variabilität der Zielgröße nicht kleiner als bei häufigen Erkrankungen sein, was die große Heterogenität des Erscheinungsbilds seltener Erkrankungen nahelegt.

Der europäische Gesetzgeber fordert also eigentlich Unmögliches, wenn er in der Verordnung der Europäischen Gemeinschaft EG Nr. 141/2000 explizit da- 
rauf hinweist, dass diese Patienten Anspruch darauf haben, mit Medikamenten behandelt zu werden, die genauso sorgfältig getestet sind wie die Arzneimittel, die zur Behandlung häufiger Erkrankungen herangezogen werden. Was kann getan werden, wenn doch die Anzahl der Patienten, die in eine klinische Studie einbezogen werden können, aufgrund der Tatsache, dass es sich um ein seltenes Leiden handelt, sehr begrenzt ist?

Betrachtet man dieses Problem differenzierter, so erkennt man, dass es unterhalb der Prävalenzgrenze von 5/10.000 Einwohnern der Europäischen Union auch recht häufige seltene Erkrankungen wie die zystische Fibrose gibt, bei der es durchaus möglich ist, neue Arzneimittel oder neue Darreichungsformen von Arzneimitteln in Studien mit rund 500 Patienten zu untersuchen und damit eine Datenbasis für die Zulassung der Arzneimittel zu schaffen, die konventionellen Arzneimittelentwicklungen in nichts nachstehen. Die zur Behandlung dieser Erkrankung grundlegend erforderlichen Antibiotika werden bezüglich ihrer prinzipiellen Wirksamkeit und Sicherheit auch in anderen, nichtseltenen Indikationen untersucht und folglich muss an vielen Stellen nur die Übertragbarkeit der Ergebnisse und der besondere Nutzen für die speziellen Gegebenheiten der Erkrankung zystische Fibrose untersucht werden.

Neben den häufigeren seltenen Erkrankungen gibt es jedoch zahlreiche $\mathrm{Ab}$ stufungen von selten, wobei Morbus Gaucher mit im Mittel 0,06 Erkrankten unter 10.000 Einwohnern eine der häufigeren lyosomalen Speichererkrankungen ist. Für die seltensten finden sich innerhalb der Europäischen Union dagegen nur weniger als 50-100 Patienten.

Es sind die Erkrankungen dieser letzten Gruppen, die aus methodischer und erkenntnislogischer Sicht besondere Herausforderungen stellen. Aus methodischer Sicht interessiert, ob ein langsamer Krankheitsverlauf positiv zu beeinflussen ist (und ein Patient ggf. bei begrenztem Risiko mehrfach in klinischen Studien behandelt wird und zum wissenschaftlichen Erkenntnisgewinn beitragen könnte) oder ob ein terminales Ereignis verhindert werden soll.
In seltenen Fällen stehen Surrogatzielgrößen zur Verfügung, deren positive Beeinflussung auf die Wirksamkeit des Arzneimittels schließen lassen. Zu beachten ist jedoch, dass die Validierung einer Surrogatzielgröße ein eigenständiges Forschungsprojekt ist, für das auch Ressourcen und Patienten benötigt werden. Vergleichbares gilt für die Untersuchung von Biomarkern.

In den letzten Jahren ist eine Reihe von methodischen Arbeiten publiziert worden, die für die Nutzung der klassischen Studiendesigns im Bereich seltener Erkrankungen argumentieren und dabei, so ist kritisch anzumerken, nur die generelle Eignung verschiedener Designs in bestimmten Anwendungssituationen diskutieren, ohne auf die spezielle Eignung für den Fall seltener Erkrankungen einzugehen [6]. Als Beispiel mag die regelhafte Empfehlung eines Parallelgruppenvergleichs mit verzögertem Therapiebeginn in einem Studienarm im Vergleich zum sofortigen Studienbeginn im anderen Arm dienen. Häufig wird hervorgehoben, dass auf diesem Wege die Zeit unter Placebo verkürzt wird, was dann als Vorteil zu werten wäre, wenn denn die experimentelle Therapie wirksam und sicher wäre. Nicht diskutiert wird, dass das sogenannte „delayed start design“ gleichzeitig zur Beobachtung eines reduzierten Therapieeffekts führt, der folglich, um ein gleiches Signifikanzniveau zu erreichen, durch eine größere Fallzahl ausgeglichen werden muss, was im Bereich seltener Erkrankungen ein eher kontraproduktiver Ansatz ist. Weiterführende Überlegungen, dass mit diesem Ansatz dann auch für eine Nutzen-Risiko-Bewertung der Nutzen nicht korrekt bemessen werden kann, sondern lediglich ein formaler Wirksamkeitsnachweis geführt wird, bleiben ebenfalls unerwähnt.

Die Entwicklung neuer Methoden, mit deren Hilfe eine Reduktion der erforderlichen Fallzahl möglich wäre, würde auch der Forschung an häufigen Erkrankungen zu Gute kommen. Denn auch dort sollten nicht mehr Patienten mit einer unterlegenen Therapie behandelt werden als erforderlich, um den Nachweis der Wirksamkeit und eines positiven Nutzen-Risiko-Verhältnisses zu begründen.
Wenn Größe und Variabilität des erwarteten Therapieeffekts und nicht die Häufigkeit der Erkrankung die erforderliche Fallzahl bestimmen, stellt sich die Frage, wie sich die Prüfung von Arzneimitteln für seltene Erkrankungen von der für häufige Erkrankungen unterscheiden könnte. So könnte man theoretisch zum Zeitpunkt der Zulassung eines Arzneimittels für eine seltene Erkrankung größere Risiken, z. B. durch die Erhöhung des Fehlers 1. Art von $5 \%$ auf $10 \%$ in Kauf nehmen, was zu einer Reduktion der Fallzahlanforderung führt. Dann müsste es aber eine nachvollziehbare und implementierte Strategie geben, wie man nach der Zulassung die noch fehlende Evidenz beibringen kann. Eine weitere Studie zur Untersuchung der Wirksamkeit nach der Zulassung des Arzneimittels (eine sog. „post-authorization efficacy study “ [PAES]) wäre eine Möglichkeit, die in ihrer praktischen Umsetzung aber auf viele Probleme stößt: Nichtrandomisierte Studien haben eine begrenzte Aussagekraft, randomisierte (und dann speziell placebokontrollierte) Studien sind für mögliche Prüfungsteilnehmer wenig attraktiv. Wer würde an einem Experiment zur Untersuchung eines Arzneimittels teilnehmen wollen, für das bereits so viel Evidenz vorliegt, dass eine Zulassung ausgesprochen werden könnte? Rechtliche Probleme, die Zulassung eines Arzneimittels zu widerrufen, bestehen, wenn Studien nach Zulassung nicht oder nicht erfolgreich abgeschlossen werden. Ohne derartige Überlegungen wird man jedoch dem gesetzlichen Auftrag nicht gerecht, wenn man einfach die Wahrscheinlichkeit für den Fehler 1. Art vergrößert.

Wir empfehlen, dass stets zunächst eine Fallzahlplanung für ein klassisches Parallelgruppendesign (oder ein anderes Standarddesign) vorgelegt wird. Bestehen Designoptionen oder alternative methodische Ansätze („delayed start designs“, Cross-Over-Studien, adaptive Designs, Bayes-Verfahren) kann dann mit biometrischen Methoden abgeschätzt werden, welche Fallzahlreduktion unter welchen Zusatzannahmen möglich ist, aber auch welche Annahmen der entsprechenden Verfahren bezüglich ihrer Validität bevorzugt aus externen Informationen, notfalls aber auch aus 
den Daten selbst, plausibel begründet werden müssen.

Konfirmatorische Studien zeichnen sich dadurch aus, dass präspezifizierte Hypothesen nach Durchführung einer experimentellen Studie geprüft werden. Dieses Vorgehen hat eine hohe Ergebnissicherheit und hat die Therapieforschung vorangebracht. Erste Ansätze dazu, wie Studien unter Einhaltung der Standards so geplant werden können, dass im Falle einer Missspezifikation oder eben nicht ausreichender Fallzahl trotzdem Entscheidungen unter Einhaltung einer Irrtumswahrscheinlichkeit getroffen werden können, liegen vor [7]. Sie können als Modell für Ansätze dienen, bei denen nicht einfach der Fehler 1. Art erhöht wird, sondern Standards beibehalten und „Fallbackpositionen“ entwickelt werden, die greifen, wenn eine Einhaltung des Standards nicht möglich ist, und es erlauben trotzdem präspezifizierte Hypothesen unter Einhaltung einer Irrtumswahrscheinlichkeit zu bewerten. So ist in der vorgenannten Arbeit aufgezeigt worden wie in einer Situation, in der üblicherweise der Behandlungseffekt mit zwei koprimären Endpunkten beschrieben wird, ggf. unter Einhaltung einer Irrtumswahrscheinlichkeit eine konfirmatorische Entscheidung getroffen werden kann, wenn nur einer der beiden Endpunkte ein „signifikant“ erreicht hat. Andere Ansätze sind denkbar, in denen zunächst eine Minimalanforderung geprüft wird (z. B. Schaden kann ausgeschlossen werden) bevor dann ein positiver Effekt nachgewiesen werden soll. Falls ein formaler Wirksamkeitsnachweis in der zweiten Stufe scheitern würde, wäre man auf Basis der gezeigten Minimalanforderung sicher in einer besseren Position, Nutzen und Risiken der Behandlung einzuschätzen.

Der gesetzliche Auftrag der Arzneimittelzulassung ist es, den formalen Nachweis der Wirksamkeit zu prüfen und das Vorliegen eines positiven Nutzen-Risiko-Verhältnisses festzustellen. Man könnte die Auffassung vertreten, dass dem zweiten Schritt im Bereich der Zulassung von Arzneimitteln für seltene Leiden eine größere Bedeutung zukommt, jedoch sollte die Bedeutung eines formalen Wirksamkeitsnachwei- ses nicht unterschätzt werden, da er die prinzipielle Voraussetzung dafür ist, eine Entscheidung darüber herbeizuführen, ob ein Therapieeffekt eine Zufallsschwankung oder ein systematischer Effekt ist.

\section{Gesichtspunkte für Ethik- Kommissionen}

Im Folgenden haben wir eine Reihe von Fragen und Überlegungen zusammengestellt, die in diesem Zusammenhang im Studienprotokoll adressiert sein sollten:

1. Verspricht die Studie nichts, was sie nicht auch halten kann?

Nicht nur im Bereich seltener Erkrankungen, dort aber ganz besonders, besteht eine gewisse Gefahr, „die Dinge passend zu machen" und z. B. einen Therapieeffekt anzunehmen, der unwahrscheinlich groß ist, aber zu einem „realisierbaren“ Stichprobenumfang führt. Dies ist der Sache nicht dienlich, denn Wunder sind selten und die Studie führt dann meist zu einem nichtsignifikanten Ergebnis. Lieber eine klare Analyse dessen, was unter Berücksichtigung klinisch-pharmakologischer Überlegungen plausibel ist. Als Korollar ergibt sich, dass die Wirksamkeit unter dem gegenwärtigen Standard korrekt beschrieben werden sollte und dafür hinreichende Information in Form eines systematischen Reviews bereitgestellt wird.

2. Ist die Studie informativ, auch wenn die experimentelle Therapie nicht erfolgreich ist?

Die Studienplanung unter der Annahme sehr überschätzter Effekte hat auch den Nachteil, dass in dem Fall, in dem die Studie ihr primäres Ziel nicht zeigen kann, dennoch das Vorhandensein starker Effekte nicht ausgeschlossen werden kann. Seit jeher empfehlen Biometriker daher, Studien für einen klinisch relevanten Effekt in der Indikation zu planen und nicht für den erhofften Effekt. Man sollte also bereits zum Zeitpunkt der Planung überlegen, wie man die Studienhypothese auf der Basis des zugehörigen Konfidenzintervalls bewerten würde, wenn das Ergebnis nicht signifikant wäre und sich
Gedanken darüber machen, ob der Therapieeffekt hinreichend genau geschätzt wird, sodass unrealistische Effekte ausgeschlossen werden können. Eine Studie muss wenigstens so groß geplant werden, dass die Forschungsfragestellung ad acta gelegt werden kann, wenn das Studienergebnis die Studienhypothese nicht stützt.

3. Keine Pilotstudien

Häufig wird als Begründung für eine unzureichende Fallzahlallokation für eine Studie angeführt, es handele sich „lediglich“ um eine Pilotstudie, auf die dann, falls erfolgreich, eine konfirmatorische Studie folgen würde. Im Bereich der seltenen Erkrankungen sprechen mehrere Argumente dagegen, diesem Argument zu folgen: Erstens ist zu erwarten, dass die Studie eine lange Laufzeit haben wird. Falls sie denn erfolgreich abgeschlossen ist, wird ein beachtlicher Druck bestehen, die Ergebnisse unverzüglich zur Anwendung zu bringen. Zu diesem Zeitpunkt eine randomisierte Studie mit einer deutlich größeren Fallzahl und entsprechend längeren Laufzeit zu planen, um die Ergebnisse zu bestätigen, dürfte kaum jemals umsetzbar sein. Zweitens wird man eine weitere Studie nur dann in Erwägung ziehen, wenn die Pilotstudie ein eindeutig vielversprechendes Ergebnis gezeigt hat. Das erhöht den Druck, die Therapie direkt einzusetzen und schafft ein ethisches Problem: Randomisieren darf man nur, wenn in der Wissenschaftsgemeinde Unklarheit darüber herrscht, ob eine Therapie überlegen wirksam ist. Eine erfolgreiche Pilotstudie kann diese Annahme der Nullhypothese, oft auch „Equipoise“ genannt, problematisch werden lassen und zugleich Ärzte und Patienten davon abhalten, an einer Folgestudie teilzunehmen, da die dann vorliegende (aus statistischer Sicht ggf. schwache) Evidenz manchem genügen mag, einen Heilversuch zu unternehmen. Um dieses ethische Dilemma zu vermeiden, wurde schon 1977 empfohlen, bereits den ersten Patienten in einer randomisierten Studie zu behan- 
deln [8]. Auch wenn dies eine sehr extreme Vorstellung ist, ist das Denkmodell hilfreich, sich die negativen Auswirkungen der Durchführung einer unzureichenden Pilotstudie vor Augen zu führen. In der Summe bleibt, dass die vorliegende Pilotstudie mit großer Wahrscheinlichkeit die einzige Studie ist, die zu dieser Fragestellung durchgeführt wird, da es fast immer mehr Ideen dazu gibt, wie Patienten behandelt werden könnten, als Studien durchgeführt werden können. Falls die Ergebnisse also nicht besonders erfolgversprechend erscheinen, wird vermutlich eher ein anderes Therapiekonzept untersucht werden, als den bisherigen Forschungsansatz zu einem eventuell durchaus erfolgreichen Abschluss weiter zu verfolgen.

Alle vorgenannten Argumente sprechen dafür, von Anfang an eine angemessene Fallzahl zu wählen, die es erlaubt, den Behandlungseffekt mit einer vernünftigen Präzision zu schätzen und zugleich eine NutzenRisiko-Abwägung durchzuführen. Die initial von Chalmers [8] erhobene Forderung, bereits den ersten Patienten zu randomisieren, sollte wenigstens in der abgeschwächten Form, jeden Patienten im Rahmen einer (zielführenden) Studie zu behandeln, speziell in der Situation seltener Leiden, nachdrücklich vertreten werden, um so den Erkenntnisgewinn in einer schwierigen Situation voranzutreiben.

4. Wie wird sichergestellt, dass die Studie beendet werden kann, wenn sie ihr Ziel nicht erreicht? Jede Studie im Bereich seltener Erkrankungen sollte von einem kompetenten Data Monitoring Committee bzw. einem Data Safety Monitoring Board (DSMB) begleitet werden. Neben der üblichen Aufgabe die Sicherheit der Patienten im Studienverlauf zu überwachen sollte das DSMB auch die Kompetenz besitzen, rationale Entscheidungen zu treffen, ob eine Weiterführung der Studie sinnhaft ist oder ob aus der gemeinsamen Bewertung von primären und sekundären Zielgrö- ßen geschlossen werden kann, dass relevante Therapieeffekte und ein positives Nutzen-Risiko-Verhältnis nicht gezeigt werden können. Wenn nur wenige Patienten für die Therapieforschung zur Verfügung stehen, muss auch überlegt werden, ob die Studie nicht besser beendet wird, sodass diese Patienten für die Untersuchung anderer Therapieansätze zur Verfügung stehen. Wichtig ist, dass die Entscheidung über Fortführung oder Abbruch einer klinischen Prüfung auf der Basis der Ergebnisse der aktuellen Studie geführt wird (die ggf. durch statistische Methoden wie das sogenannte Stochastic-Curtailment- bzw. Conditional-PowerVerfahren unterstützt werden kann). Diese Entscheidung sollte jedoch nicht miteinbeziehen, ob es andere, möglicherweise vielversprechendere, aber zu diesem Zeitpunkt schlechter erprobte Therapiekonzepte gibt: Die Fortführung einer begonnenen Studie ist ein ethisches Mandat, solange sich aus der aktuellen Studie keine Ergebnisse ableiten lassen, die eine andere Entscheidung nahelegen. Aus diesem Blickwinkel wird die Wichtigkeit einer qualitativ hochwertigen Studienplanung sofort deutlich: Kontrolle, Randomisierung und ggf. Verblindung vermeiden Patientenselektion und führen zu einer unverzerrten und somit besten Schätzung des Therapieeffekts in einer Studie. Einarmige Studien unterliegen dem Risiko, dass beobachtete Therapieerfolge größtenteils auch der erfolgreichen Selektion von Patienten zugeschrieben werden könnten. Offensichtlich ist eine Entscheidung darüber, ob der Therapieeffekt relevant ist oder nicht, nur dann möglich, wenn Verzerrungen der Schätzung des Therapieeffekts ausgeschlossen werden können.

Die Zulassungsbehörden und EthikKommissionen beteiligen sich essenziell an der Verantwortung für die korrekte Prüfung der Wirksamkeit und Sicherheit eines Arzneimittels. BfArM, PEI und EMA stehen zudem im Vorfeld zur Verfügung, Mindestanforderungen für eine positive
Zulassungsentscheidung festzulegen. Für nichtmedikamentöse Verfahren gibt es außer den Ethikkommissionen kein wirkliches institutionalisiertes Gremium zur Beratung eines Forschers zu Fragen der Studienplanung. Hier kommt den Ethikkommissionen die Rolle des kritischen Gegenübers zu, der die Überlegungen des Forschers unabhängig prüft und im Sinne der externen wissenschaftlichen Bewertung vor Beginn der Studie die Machbarkeit und die Durchführbarkeit konstruktiv kritisch begleitet. Die Autoren vertreten die Auffassung, dass diese Diskussionen um das Design einer Studie einen ganz wesentlichen Beitrag dazu leisten, dass Therapiestudien optimal, zielführend und erfolgreich durchgeführt werden und zugleich wesentliche ethische Anforderungen erfüllen.

Wie gezeigt lassen sich für die Bewertung von klinischen Prüfungen für seltene Erkrankungen Abstriche bei der ethischen Bewertung nicht begründen. Auch die Generierung aussagekräftigen Wissens zu Wirksamkeit und Sicherheit für eine evidenzbasierte medizinische Versorgung seltener Erkrankungen stößt auf methodische Grenzen. Es bleibt zu hoffen, dass die gegenwärtig von der EU geförderten Forschungsverbünde zu „clinical trials in rare diseases “ neue und tragfähige Optionen entwickeln [9].

\section{Fazit für die Praxis}

Weder aus ethischer noch aus rechtlicher Sicht gibt es eine Begründung, unterschiedliche Anforderungen an die Prüfung von Therapien für seltene und häufige Leiden zu stellen. Offensichtlich ist die Anzahl der Patienten, die in eine Prüfung einbezogen werden können, begrenzt. Aus methodischer Sicht gilt es deshalb, die Studie bestmöglich so zu planen, dass, auch wenn vielleicht keine "Signifikanz" erreicht werden kann, die Schätzung des Therapieeffekts unverzerrt ist und Nutzen und Risiken zu einer Kontrolltherapie in Beziehung gesetzt werden können. 


\section{Korrespondenzadresse}

\section{Prof. Dr. J. Hasford}

Institut für Medizinische Informationsverarbeitung, Biometrie und Epidemiologie, Ludwig-

Maximilians-Universität München

Marchioninistr. 15, 81377 München,

Deutschland

has@ibe.med.uni-muenchen.de

Danksagung. Die Autoren danken den Gutachtern für die konstruktiven Hinweise zu den Überlegungen des Papers. Die Arbeit von Herrn Koch wird teilweise unterstützt durch das FP7-HEALTH-2013INNOVATION-1-Projekt „Advances in Small Trials Design for Regulatory Innovation and Excellence" (ASTERIX) Grant Agreement No. 603160.

\section{Einhaltung ethischer Richtlinien}

Interessenkonflikt. J. Hasford und A. Koch geben an, dass kein Interessenkonflikt besteht.

Dieser Beitrag beinhaltet keine von den Autoren durchgeführten Studien an Menschen oder Tieren.

\section{Literatur}

1. Nationales Aktionsbündnis für Menschen mit Seltenen Erkrankungen (2013) Nationaler Aktionsplan für Menschen mit Seltenen Erkrankungen. Nationales Aktionsbündnis für Menschen mit Seltenen Erkrankungen, Bonn

2. Beauchamp TL, Childress JF (2013) Principles of biomedical ethics, 7. Aufl. Oxford University Press, New York

3. Bolignano D, Pisano A (2016) Good-quality research in rare diseases: trials and tribulations. Pediatr Nephrol 31:2017-2023

4. Gagne JJ, Thompson L, O'Keefe K, Kesselheim AS (2014) Innovative research methods for studying treatments for rare diseases: methodological review. BMJ 349:g6802

5. EMEA (2007) Guideline on clinical trials in small populations. http://www.ema.europa.eu/docs/ en_GB/document_library/Scientific_guideline/ 2009/09/WC500003615.pdf. Zugegriffen: 03. Febr 2017

6. Cornu C, Kassai B, Fisch R et al (2013) Experimental designs for small randomised clinical trials: an algorithm for choice. Orphanet J Rare Dis 8:48

7. Ristl R, Frommlet F, Koch A, Posch M (2016) Fallback tests for co-primary endpoints. Stat Med 35:2669-2686

8. Chalmers TC (1977) Randomize the first patient. NEngl J Med 296:107

9. http://ec.europa.eu/research/health/index.cfm? pg=area\&areaname=rare. Zugegriffen: 04. Februar 2017 